\title{
On the generalized Burgers equation
}

\author{
Alkis S. Tersenov
}

\begin{abstract}
The present paper is concerned with the initial boundary value problem for the generalized Burgers equation $u_{t}+g(t, u) u_{x}+f(t, u)=$ $\varepsilon u_{x x}$ which arises in many applications. We formulate a condition guaranteeing the a priori estimate of $\max \left|u_{x}\right|$ independent of $\varepsilon$ and $t$ and give an example demonstrating the optimality of this condition. Based on this estimate we prove the global existence of a unique classical solution of the problem and investigate the behavior of this solution for $\varepsilon \rightarrow 0$ and $t \rightarrow+\infty$. The Cauchy problem for this equation is considered as well.
\end{abstract}

Mathematics Subject Classification (2000). 35K55, 35K60, 35F30, 35F25.

Keywords. Semilinear parabolic equations, Boundary value problems, A priori estimates, Global solvability.

\section{Introduction and main results}

Consider the following equation

$$
u_{t}+g(t, u) u_{x}+f(t, u)=\varepsilon u_{x x} \quad \text { in } Q_{T}=(0, T) \times(-l, l),
$$

coupled with initial condition

$$
u(0, x)=\phi(x) \text { for } x \in[-l, l],
$$

and boundary conditions

$$
u(t, \pm l)=0 \text { for } t \in(0, T),
$$

with arbitrary $T>0$. Here $\varepsilon$ is a positive constant, $f(t, z), g(t, z)$ and $\phi(x)$ are given functions. Equation (1.1) appears in different applications, see [2,6$13,16,17]$. The goal of the present paper is to obtain an a priori estimate of $\left|u_{x}\right|$ independent of $\varepsilon$ and $T$ and on the basis of this estimate to prove the existence and the uniqueness of the classical solution of problem (1.1)-(1.3) and to investigate the behavior of this solution when $\varepsilon \rightarrow 0$ and when $T \rightarrow+\infty$. Let us mention that in [14] an a priori estimate of the gradient of a solution of fully nonlinear parabolic equation independent on $\varepsilon$ and $T$ was obtained under several restrictions (see assumptions $(0.10)-(0.12)$ in [14]). One can easily see 
that these restrictions are not fulfilled in our case. However more delicate analysis shows that we can extend approach developed in [14] to Eq. (1.1) as well. Again (as in [14]) we apply the modification of the very effective and fruitful Kruzhkov's idea of introducing of an additional spatial variable [4].

Condition guaranteeing the needed a priori estimate is the following one:

$$
K\left|g\left(t, u_{2}\right)-g\left(t, u_{1}\right)\right|<f\left(t, u_{2}\right)-f\left(t, u_{1}\right) \quad \text { for }-M \leq u_{1}<u_{2} \leq M,
$$

where

$$
M=\sup _{[-l, l]}|\phi(x)|, \quad K=\sup _{x, y \in[-l, l]} \frac{|\phi(x)-\phi(y)|}{|x-y|} .
$$

This is the only structure restriction on $g$ and $f$ and as it follows from Example 1 below, this condition cannot be improved.

Consider the limit case $(\varepsilon=0)$

$$
\begin{aligned}
v_{t}+g(t, v) v_{x}+f(t, v) & =0 \quad \text { in } Q_{T}=(0, T) \times(-l, l), \\
v(0, x) & =\phi(x) \quad \text { for } x \in[-l, l],
\end{aligned}
$$

and

$$
v(t, \pm l)=0 \quad \text { for } t \in(0, T) .
$$

Definition 1. We say that $u(t, x)$ is a classical solution of problem (1.1)-(1.3) if $u(t, x) \in C_{t, x}^{1+\alpha / 2,2+\alpha}\left(Q_{T}\right) \cap C_{t, x}^{1 / 2,1}\left(\bar{Q}_{T}\right)$ for some $\alpha \in(0,1)$ and it satisfies Eq. (1.1) pointwise.

We say that function $v(t, x)$ is a strong solution of problem (1.5)-(1.7) if $v(t, x)$ is Lipschitz continuous and satisfies Eq. (1.5) almost everywhere (a.e.).

Theorem 1. Suppose that $f(t, z)$ and $g(t, z)$ are Hölder continuous functions on the set $[0, T] \times[-M, M], \phi(x)$ is Lipschitz continuous function on $[-l, l]$ and $f(t, 0)=g(t, 0)=\phi( \pm l)=0$. If condition (1.4) is fulfilled then

I. there exists a unique classical solution of problem (1.1)-(1.3) and

$$
\max _{Q_{T}}|u(t, x)| \leq M, \quad \max _{Q_{T}}\left|u_{x}(t, x)\right| \leq K,
$$

moreover

$$
u(t, x) \rightarrow 0 \quad \text { when } t \rightarrow+\infty .
$$

II. there exists a strong solution of problem (1.5)-(1.7) such that

$$
\max _{Q_{T}}|v(t, x)| \leq M, \quad\left\|v_{x}(t, x)\right\|_{L_{\infty}} \leq K,
$$

moreover if $g(t, v)$ is differentiable with respect to $v$ this solution is unique and

$$
v(t, x) \rightarrow 0 \quad \text { when } t \rightarrow+\infty
$$

III.

$$
\lim _{\varepsilon \rightarrow 0}\|u(t, x)-v(t, x)\|_{C^{0}\left(Q_{T}\right)}=0 .
$$


Note that for the uniqueness of problem (1.1)-(1.3) we do not require Lipschitz continuity of $f(t, z)$ and $g(t, z)$ with respect to $z$.

Example 1. The simplest case of (1.1) is the following equation (see $[6-13,16])$

$$
u_{t}+a u u_{x}+\lambda u=\varepsilon u_{x x}
$$

where $\lambda>0$ and $a$ are constants.

We have $g(t, u)=a u$ and $f(t, u)=\lambda u$. Condition (1.4) takes the form

$$
\lambda>|a| K
$$

(concerning the case $\lambda \leq|a| K$ see Sect. 5 of the present paper). Let us show that condition (1.9) is optimal. Consider the limit case

$$
v_{t}+a v v_{x}=-\lambda v \text {. }
$$

According to Theorem 1, condition (1.9) provides the existence of the global classical solution of problem (1.8), (1.2), (1.3) and as a consequence the existence of the global strong solution of problem $(1.10),(1.6),(1.7)$. It is well known that, even for smooth initial data, the solution of problem (1.5)-(1.7) can develop shocks in finite time. Global Lipschitz continuous solution of this problem exists only if characteristics do not intersect. The family of characteristics $x=x(t)$ is defined by

$$
x(t)=x(0)+\frac{a}{\lambda} \phi(x(0))\left(1-e^{-\lambda t}\right) .
$$

Suppose that the characteristics $x_{1}(t)$ and $x_{2}(t)$ which start from the points $x_{1}(0)$ and $x_{2}(0)$ respectively intersect at time $t^{*}>0$ i. e. $x_{1}\left(t^{*}\right)=x_{2}\left(t^{*}\right)$ or

$$
x_{1}(0)+\frac{a}{\lambda} \phi\left(x_{1}(0)\right)\left(1-e^{-\lambda t^{*}}\right)=x_{2}(0)+\frac{a}{\lambda} \phi\left(x_{2}(0)\right)\left(1-e^{-\lambda t^{*}}\right)
$$

or

$$
\frac{\left|\phi\left(x_{1}(0)\right)-\phi\left(x_{2}(0)\right)\right|}{\left|x_{1}(0)-x_{2}(0)\right|}=\frac{\lambda}{|a|} \frac{e^{\lambda t^{*}}}{e^{\lambda t^{*}}-1} .
$$

The last is possible only if $\lambda \leq|a| K$, hence condition $\lambda>|a| K$ is optimal. Here we use the obvious inequality

$$
\frac{e^{\lambda t^{*}}}{e^{\lambda t^{*}}-1} \geq 1 \quad \text { for } t^{*} \geq 0 .
$$

Example 2. Consider equation

$$
u_{t}+a u^{2} u_{x}+\lambda u=\varepsilon u_{x x}
$$

(see, for example, $[11,13])$. Condition (1.4) takes the form

$$
K|a|\left|u_{2}+u_{1}\right|<\lambda \text {. }
$$

Taking into account that for the solution of problem (1.11), (1.2), (1.3) the estimate

$$
|u(t, x)| \leq M
$$

holds, we conclude that (1.4) is fulfilled if

$$
\lambda>2 K|a| M \text {. }
$$


Example 3. Consider equation

$$
u_{t}+a u^{2 / 3} u_{x}+\lambda u^{1 / 3}=\varepsilon u_{x x}
$$

(see $[11,13])$. Condition (1.4) takes the form

$$
K|a|\left|u_{2}^{1 / 3}+u_{1}^{1 / 3}\right|<\lambda .
$$

Taking into account that for the solution of problem (1.12), (1.2), (1.3) as in the previous example we have $|u(t, x)| \leq M$ we conclude that (1.4) is fulfilled if

$$
\lambda>2 K|a| M^{1 / 3}
$$

One can easily construct other examples of functions $f$ and $g$ satisfying condition (1.4), in particular if $f=K_{0} g$ with constant $K_{0}>K$ then (1.4) is obviously fulfilled.

The paper is organized as follows. In Sect. 2 we obtain the a priori estimate of the gradient of the solution. In Sect. 3 we prove Theorem 1. In Sect. 4 we extend our results to the Cauchy problem and in the last Section we discuss Eq. (1.8) for which condition (1.9) is not fulfilled.

\section{A priori estimate of $\boldsymbol{u}_{\boldsymbol{x}}$}

Lemma 1.1. Suppose that condition (1.4) is fulfilled and $f(t, 0)=g(t, 0)=$ $\phi( \pm l)=0$, then for any classical solution of problem (1.1)-(1.3) the estimate

$$
\left|u_{x}(t, x)\right| \leq K \quad \text { for all } t \geq 0
$$

holds.

Proof. Consider Eq. (1.1) in two different points $(t, x)$ and $(t, y)$ :

$$
\begin{aligned}
& u_{t}(t, x)-\varepsilon u_{x x}(t, x)=-f(t, u(t, x))-g(t, u(t, x)) u_{x}(t, x), \\
& u_{t}(t, y)-\varepsilon u_{y y}(t, y)=-f(t, u(t, y))-g(t, u(t, y)) u_{y}(t, y) .
\end{aligned}
$$

Subtracting Eq. (2.2) from (2.1) for the function

$$
w(t, x, y) \equiv u(t, x)-u(t, y)-K(x-y)
$$

we obtain

$$
\begin{aligned}
& w_{t}-\varepsilon w_{x x}-\varepsilon w_{y y} \\
& \quad=-[f(t, u(t, x))-f(t, u(t, y))]-\left[g(t, u(t, x)) u_{x}(t, x)-g(t, u(t, y)) u_{y}(t, y)\right] .
\end{aligned}
$$

Consider Eq. (2.3) in

$$
P=\{0<t \leq T,-l<x, y<l, y<x\} .
$$

Suppose that the function $w$ attains its positive maximum at some point $N \in$ $P$, then at this point we have

$$
w>0, \quad w_{x}=w_{y}=0, \quad \text { i.e. } u(t, x)>u(t, y), \quad u_{x}(t, x)=u_{y}(t, y)=K
$$


and

$$
\begin{aligned}
w_{t} & -\varepsilon w_{x x}-\left.\varepsilon w_{y y}\right|_{N} \\
& =-[f(t, u(t, x))-f(t, u(t, y))]-\left.K[g(t, u(t, x))-g(t, u(t, y))]\right|_{N} .
\end{aligned}
$$

Hence due to (1.4)

$$
w_{t}-\varepsilon w_{x x}-\left.\varepsilon w_{y y}\right|_{N}<0
$$

which is impossible.

Denote by $\Gamma$ the parabolic boundary of $P$

$$
\Gamma=\{t=0, y \leq x,-l \leq x, y \leq l\} \cup\{0<t \leq T, x=y,|x|<l\} \cup \Omega_{1} \cup \Omega_{2}
$$

where

$$
\begin{aligned}
& \Omega_{1}=\{0<t \leq T,|x| \leq l, y=-l\}, \\
& \Omega_{2}=\{0<t \leq T,|y| \leq l, x=l\} .
\end{aligned}
$$

Our goal is to show that $w \leq 0$ on $\Gamma$. For $t=0$ we have

$$
w(0, x, y)=\phi(x)-\phi(y)-K(x-y) \leq 0 .
$$

For $x=y$ obviously $w=0$.

Consider $\Omega_{1}$, for $y=-l$ we have

$$
w(t, x,-l)=u(t, x)-K(x+l)
$$

and

$$
w_{t}-\left.\varepsilon w_{x x}\right|_{y=-l}=u_{t}-\varepsilon u_{x x}=-f(t, u)-g(t, u) u_{x} \quad \text { in } \Omega_{1} .
$$

Suppose that $w(t, x,-l)$ attains its positive maximum at the point $N_{1} \in \Omega_{1}$, then at this point we have

$$
w>0, \quad w_{x}=0, \quad \text { i.e. } u>0, \quad u_{x}=K
$$

and hence

$$
w_{t}-\left.\varepsilon w_{x x}\right|_{N_{1}}=-f(t, u)-\left.g(t, u) K\right|_{N_{1}} .
$$

Due to (1.4) (taking into account the assumption $f(t, 0)=g(t, 0)=0$ ) we obtain

$$
w_{t}-\left.\varepsilon w_{x x}\right|_{N_{1}}<0
$$

which is impossible. Consider the parabolic boundary of $\Omega_{1}$. If $t=0$ we have $w(0, x,-l)=\phi(x)-K(x+l) \leq 0$ due to the fact that $w(0,-l,-l)=0$ and $w_{x}(0, x,-l)=\phi^{\prime}(x)-K \leq 0$, besides $w(t, \pm l,-l) \leq 0$. Thus we conclude that $w(t, x,-l) \leq 0$ in $\bar{\Omega}_{1}$.

Similarly we investigate the sign of $w$ on $\Omega_{2}$. For $x=l$ we have

$$
w(t, l, y)=-u(t, y)-K(l-y)
$$

and

$$
w_{t}-\left.\varepsilon w_{y y}\right|_{x=l}=-u_{t}+\varepsilon u_{y y}=f(t, u)+g(t, u) u_{y} \quad \text { in } \Omega_{2} .
$$


Suppose that $w(t, l, y)$ attains its positive maximum at the point $N_{2} \in \Omega_{2}$, then at this point we have

$$
w>0, \quad w_{y}=0, \quad \text { i.e. } u<0, \quad u_{y}=K
$$

and hence [again due to (1.4)]

$$
w_{t}-\left.\varepsilon w_{y y}\right|_{N_{2}}=f(t, u)+\left.g(t, u) K\right|_{N_{2}}<0
$$

which is impossible. Consider the parabolic boundary of $\Omega_{2}$. If $t=0$ we have $w(0, l, y)=-\phi(y)-K(l-y) \leq 0$ due to the fact that $w(0, l, l)=0$ and $w_{y}(0, l, y)=-\phi^{\prime}(y)+K \geq 0$, besides $w(t, l, \pm l) \leq 0$. Thus we conclude that $w(t, l, y) \leq 0$ in $\bar{\Omega}_{2}$.

Finally, taking into account that $w(t, x, y)$ cannot attain its positive maximum in $P$ and is non positive on the parabolic boundary of $P$ we conclude that

$$
w(t, x, y) \leq 0 \quad \text { in } \bar{P} .
$$

Subtracting now (2.1) from (2.2) one can easily see that the function

$$
\tilde{w}(t, x, y) \equiv u(t, y)-u(t, x)-K(x-y)
$$

satisfies the equation

$$
\begin{aligned}
& \tilde{w}_{t}-\varepsilon \tilde{w}_{x x}-\varepsilon \tilde{w}_{y y}=-[f(t, u(t, y))-f(t, u(t, x))] \\
& \quad-\left[g(t, u(t, y)) u_{y}(t, y)-g(t, u(t, x)) u_{x}(t, x)\right] \text { in } P .
\end{aligned}
$$

Suppose that $\tilde{w}$ attains its positive maximum at the point $M \in P$, then at this point we have

$$
\tilde{w}>0, \tilde{w}_{x}=\tilde{w}_{y}=0, \quad \text { i.e. } u(t, y)>u(t, x), u_{x}(t, x)=u_{y}(t, y)=-K
$$

and

$$
\begin{aligned}
& \tilde{w}_{t}-\varepsilon \tilde{w}_{x x}-\left.\varepsilon \tilde{w}_{y y}\right|_{M}= \\
& \quad-[f(t, u(t, y))-f(t, u(t, x))]+\left.K[g(t, u(t, y))-g(t, u(t, x))]\right|_{M} .
\end{aligned}
$$

Hence, due to (1.4)

$$
\tilde{w}_{t}-\varepsilon \tilde{w}_{x x}-\left.\varepsilon \tilde{w}_{y y}\right|_{M}<0
$$

which is impossible.

Consider the parabolic boundary of $P$. For $t=0$ we have

$$
\tilde{w}(0, x, y)=\phi(y)-\phi(x)-K(x-y) \leq 0 .
$$

For $x=y$ obviously $\tilde{w}=0$. Consider $\Omega_{1}$, for $y=-l$ we have

$$
\tilde{w}(t, x,-l)=-u(t, x)-K(x+l)
$$

and

$$
\tilde{w}_{t}-\left.\varepsilon \tilde{w}_{x x}\right|_{y=-l}=-u_{t}+\varepsilon u_{x x}=f(t, u)+g(t, u) u_{x} \quad \text { in } \Omega_{1} .
$$


Suppose that $\tilde{w}(t, x,-l)$ attains its positive maximum at the point $M_{1} \in \Omega_{1}$, then at this point we have

$$
\tilde{w}>0, \tilde{w}_{x}=0, \quad \text { i.e. } u<0, \quad u_{x}=-K
$$

and hence

$$
\tilde{w}_{t}-\left.\varepsilon \tilde{w}_{x x}\right|_{M_{1}}=f(t, u)-\left.g(t, u) K\right|_{M_{1}}<0
$$

which is impossible (the last inequality follows from (1.4) if we put $u_{2}=0$ ). Consider the parabolic boundary of $\Omega_{1}$. If $t=0$ we have $\tilde{w}(0, x,-l)=-\phi(x)-$ $K(x+l) \leq 0$ due to the fact that $\tilde{w}(0,-l,-l)=0$ and $w_{x}(0, x,-l)=-\phi^{\prime}(x)-$ $K \leq 0$, besides for $\tilde{w}(t, \pm l,-l) \leq 0$. Thus we conclude that $\tilde{w}(t, x,-l) \leq 0$ in $\bar{\Omega}_{1}$.

Similarly we investigate the sign of $\tilde{w}$ in $\Omega_{2}$, for $x=l$ we have

$$
\tilde{w}(t, l, y)=u(t, y)-K(l-y)
$$

and

$$
\tilde{w}_{t}-\left.\varepsilon \tilde{w}_{y y}\right|_{x=l}=u_{t}-\varepsilon u_{y y}=-f(t, u)-g(t, u) u_{y} \quad \text { in } \Omega_{2} .
$$

Suppose that $\tilde{w}(t, l, y)$ attains its positive maximum at the point $M_{2} \in \Omega_{2}$, then at this point we have

$$
\tilde{w}>0, \tilde{w}_{y}=0, \quad \text { i.e. } u>0, u_{y}=-K,
$$

hence

$$
\tilde{w}_{t}-\left.\varepsilon \tilde{w}_{y y}\right|_{M_{2}}=-f(t, u)+\left.g(t, u) K\right|_{M_{2}}<0
$$

which is impossible. Consider the parabolic boundary of $\Omega_{2}$. If $t=0$ we have $\tilde{w}(0, l, y)=\phi(y)-K(l-y) \leq 0$ due to the fact that $\tilde{w}(0, l, l)=0$ and $\tilde{w}_{y}(0, l, y)=\phi^{\prime}(y)+K \geq 0$, besides $\tilde{w}(t, l, \pm l) \leq 0$. Thus we conclude that $\tilde{w}(t, l, y) \leq 0$ in $\bar{\Omega}_{2}$.

Finally, taking into account that $\tilde{w}(t, x, y)$ cannot attain its positive maximum in $P$ and is non positive on the parabolic boundary of $P$ we conclude that

$$
\tilde{w}(t, x, y) \leq 0 \quad \text { in } \bar{P} .
$$
we have

From (2.4) and (2.5) we obtain that for $t \in[0, T],|x| \leq l,|y| \leq l, x \geq y$

$$
|u(t, x)-u(t, y)| \leq K(x-y) .
$$

In view of the symmetry of the variables $x$ and $y$ we can similarly consider the case $y>x$. As a result we conclude that for $t \in[0, T],|x| \leq l,|y| \leq l$ we have

$$
|u(t, x)-u(t, y)| \leq K|x-y|
$$

or

$$
\left|u_{x}(t, x)\right| \leq K
$$

Lemma is proved. 


\section{Proof of Theorem 1}

The existence of the classical solution of problem (1.1)-(1.3) follows immediately from the a priori estimates $|u| \leq M$ and $\left|u_{x}\right| \leq K$ (see, for example, [14]). If we suppose the fulfillment of condition (1.4) for all values of $u_{1}$ and $u_{2}$ $\left(u_{1}<u_{2}\right)$ then the estimate $|u| \leq M$ can be easily obtained by standard consideration (see, for example, [5]) taking into account the fact that $u f(u)>0$ for $u \neq 0$ (the last follows from (1.4)). In order to obtain this estimate in the case when (1.4) holds only for $-M \leq u_{1}<u_{2} \leq M$ consider the auxiliary equation

$$
u_{t}+g(t, u) u_{x}+\bar{f}_{M}(t, u)=\varepsilon u_{x x} \quad \text { in } Q_{T}=(0, T) \times(-l, l),
$$

coupled with conditions (1.2), (1.3), here

$$
\bar{f}_{M}(t, z)= \begin{cases}f(t, z), & |z| \leq M, \\ f(t, M), & z>M, \\ f(t,-M), & z<-M .\end{cases}
$$

Suppose that $u$ attains its positive maximum at the point $N \in \bar{Q}_{T} \backslash \Gamma_{T}$, where $\Gamma_{T}$ is the parabolic boundary of $Q_{T}$. At this point we have $\bar{f}_{M}>0$, in fact, for $0<u \leq M$ we have $\bar{f}_{M}(t, u)=f(t, u)>0$ due to (1.4), and for $M<u$ we have $\bar{f}_{M}(t, u)=f(t, M)>0$, hence

$$
u_{t}+g(t, u) u_{x}+\bar{f}_{M}(t, u)-\left.\varepsilon u_{x x}\right|_{N}>0,
$$

which is impossible. Taking into account that $u \leq M$ on $\Gamma_{T}$ we conclude that $u \leq M$ in $\bar{Q}_{T}$. Similarly we obtain the estimate from the below $u>-M$. From the estimate $|u| \leq M$ which holds for the solution of problem (3.1), (1.2), (1.3) it follows that Eqs. (3.1)) and (1.1) coincide and thus the estimate $|u| \leq M$ takes place for the solution of problem (1.1), (1.2), (1.3) as well.

Uniqueness. As usually suppose that there exist two solutions $u_{1}$ and $u_{2}$. For the difference $\mathrm{w} \equiv u_{2}-u_{1}$ we have

$$
\mathrm{w}_{t}+g\left(t, u_{2}\right) u_{2 x}-g\left(t, u_{1}\right) u_{1 x}+f\left(t, u_{2}\right)-f\left(t, u_{1}\right)-\varepsilon \mathrm{w}_{x x}=0 \quad \text { in } Q_{T}
$$

with zero initial and boundary conditions. Suppose that at the point $N \in$ $\bar{Q}_{T} \backslash \Gamma_{T}$ function w attains its positive maximum, at this point we have $w>0$ and $w_{x}=0$ i.e. $u_{2}>u_{1}$ and $u_{2 x}=u_{1 x}$. Thus

$$
\begin{aligned}
\mathrm{w}_{t} & +g\left(t, u_{2}\right) u_{2 x}-g\left(t, u_{1}\right) u_{1 x}+f\left(t, u_{2}\right)-f\left(t, u_{1}\right)-\left.\varepsilon \mathrm{w}_{x x}\right|_{N} \\
& =\mathrm{w}_{t}+\left(g\left(t, u_{2}\right)-g\left(t, u_{1}\right)\right) u_{2 x}+f\left(t, u_{2}\right)-f\left(t, u_{1}\right)-\left.\varepsilon \mathrm{w}_{x x}\right|_{N}=0 .
\end{aligned}
$$

Taking into account that $\left|u_{2 x}\right| \leq K$, from (1.4) we conclude that

$$
\left(g\left(t, u_{2}\right)-g\left(t, u_{1}\right)\right) u_{2 x}+f\left(t, u_{2}\right)-f\left(t, u_{1}\right)>0
$$

and hence

$$
\mathrm{w}_{t}-\left.\varepsilon \mathrm{w}_{x x}\right|_{N}<0
$$

which is impossible. 
Similarly at the point $N_{1} \in \bar{Q}_{T} \backslash \Gamma_{T}$ where w attains its negative minimum we have $u_{2}<u_{1}$ and $u_{2 x}=u_{1 x}$. Thus

$$
\mathrm{w}_{t}+\left(g\left(t, u_{2}\right)-g\left(t, u_{1}\right)\right) u_{2 x}+f\left(t, u_{2}\right)-f\left(t, u_{1}\right)-\left.\varepsilon \mathrm{w}_{x x}\right|_{N_{1}}=0 .
$$

Taking into account that $\left|u_{2 x}\right| \leq K$, from (1.4) we conclude that

$$
\left(g\left(t, u_{2}\right)-g\left(t, u_{1}\right)\right) u_{2 x}+f\left(t, u_{2}\right)-f\left(t, u_{1}\right)<0 \quad\left(\text { here } u_{2}<u_{1}\right)
$$

and hence

$$
\mathrm{w}_{t}-\left.\varepsilon \mathrm{w}_{x x}\right|_{N_{1}}>0
$$

which is impossible. Recalling that $\mathrm{w}=0$ on $\Gamma_{T}$ we conclude that $\mathrm{w} \equiv 0$ on $Q_{T}$.

Large time behavior. Multiplying Eq. (1.1) by $u$ and integrating by part we obtain

$$
\frac{1}{2} \frac{d}{d t} \int_{-l}^{l} u^{2} d x+\int_{-l}^{l}\left(g(t, u) u_{x}+f(t, u)\right) u d x+\varepsilon \int_{-l}^{l} u_{x}^{2} d x=0 .
$$

Taking into account the estimate $\left|u_{x}\right| \leq K$ one can easily see that

$$
\left(g(t, u) u_{x}+f(t, u)\right) u>0 \quad \text { for } u \neq 0 .
$$

In fact, from (1.4) taking $u_{1}=0$ we obtain (recall that $f(t, 0)=g(t, 0)=0$ )

$$
f(t, u)-K|g(t, u)|>0 \quad \text { for } u>0
$$

and taking $u_{2}=0$ we obtain

$$
f(t, u)+K|g(t, u)|<0 \quad \text { for } u<0 .
$$

Hence for $u>0$ we have

$$
g(t, u) u_{x}+f(t, u)>f(t, u)-K|g(t, u)|>0
$$

and for $u<0$ we have

$$
g(t, u) u_{x}+f(t, u)<K|g(t, u)|+f(t, u)<0 .
$$

Integrating (3.2) with respect to $t$ and taking into account (3.3) we obtain

$$
\frac{1}{2} \int_{-l}^{l} u^{2} d x+\varepsilon \int_{0}^{T} \int_{-l}^{l} u_{x}^{2} d x d t<\frac{1}{2} \int_{-l}^{l} \phi^{2} d x
$$

which implies

$$
\int_{0}^{+\infty} \int_{-l}^{l} u_{x}^{2} d x d t<\frac{M^{2} l}{\varepsilon}
$$

Consequently

$$
\lim _{t \rightarrow+\infty} \int_{-l}^{l} u_{x}^{2} d x=0
$$


hence

$$
\lim _{t \rightarrow \infty}\left|u_{x}(t, x)\right|=0 \quad \forall x \in[-l, l]
$$

and

$$
\lim _{t \rightarrow \infty}|u(t, x)|=0 \quad \forall x \in[-l, l] .
$$

Let us turn now to the second part of the theorem.

Existence. Denote the solution of problem (1.1)-(1.3) by $u_{\varepsilon}(t, x)$. Recall the following estimate (see [4] or [15]): for any classical solution of problem (1.1)-(1.3) the inequality

$$
\left|u_{\varepsilon}\left(t_{1}, x\right)-u_{\varepsilon}\left(t_{2}, x\right)\right| \leq C\left|t_{1}-t_{2}\right|^{1 / 2}
$$

holds, where the constant $C$ depends only on $M, K$ and $G_{0}=\max \left|g\left(t, u_{\varepsilon}\right)\right|$, $F_{0}=\max \left|f\left(t, u_{\varepsilon}\right)\right|$ (maximum is taken over the set $\left.(0, T) \times[-M, M]\right)$. Multiply Eq. (1.1) by $u_{t}^{\varepsilon}$ and integrate by part to obtain

$$
\int_{-l}^{l} u_{\varepsilon t}^{2} d x+\frac{\varepsilon}{2} \frac{d}{d t} \int_{-l}^{l} u_{\varepsilon x}^{2} d x=-\int_{-l}^{l}\left(g\left(t, u_{\varepsilon}\right) u_{\varepsilon x}+f\left(t, u_{\varepsilon}\right)\right) u_{\varepsilon t} d x,
$$

here we use the fact that the mixed derivative $u_{\varepsilon t x} \in L_{2}\left(Q_{T}\right)$ (see [5]). Integrating with respect to $t$ and applying Hölder and Young inequalities we obtain

$$
\int_{Q_{T}} u_{\varepsilon t}^{2} d x d t \leq \int_{Q_{T}}\left(g\left(t, u_{\varepsilon}\right) u_{\varepsilon x}+f\left(t, u_{\varepsilon}\right)\right)^{2} d x d t+\varepsilon \int_{-l}^{l} \phi^{\prime 2} d x .
$$

Our goal is to pass to the limit when $\varepsilon \rightarrow 0$ hence, without loss of generality we can take $\varepsilon \leq 1$ and from the previous inequality conclude that

$$
\int_{Q_{T}} u_{\varepsilon t}^{2} d x d t \leq 2 l T\left(G_{0} K+F_{0}\right)^{2}+2 l K^{2} .
$$

Taking into account the estimates $\left|u_{\varepsilon}\right| \leq M,\left|u_{\varepsilon x}\right| \leq K$ and (3.4), (3.5) we conclude that there exists a subsequence $\varepsilon_{n}$ such that when $n \rightarrow \infty\left(\varepsilon_{n} \rightarrow 0\right)$ :

$$
\begin{array}{ll}
u_{\varepsilon_{n}} \rightarrow v & \text { uniformly, } \\
u_{\varepsilon_{n} x} \rightarrow v_{x} & { }^{*} \text { weakly in } L_{\infty}\left(Q_{T}\right), \\
u_{\varepsilon_{n}} \rightarrow v_{t} & \text { weakly in } L_{2}\left(Q_{T}\right) .
\end{array}
$$

Consequently, we can pass to the limit in the identity

$$
\begin{aligned}
& \int_{Q_{T}}\left[u_{\varepsilon_{n} t}+g\left(t, u_{\varepsilon_{n}}\right) u_{\varepsilon_{n} x}+f\left(t, u_{\varepsilon_{n}}\right)\right] \psi d x d t \\
& \quad=-\varepsilon \int_{Q_{T}} u_{\varepsilon_{n} x} \psi_{x} d x d t
\end{aligned}
$$

and obtain

$$
\int_{Q_{T}}\left[v_{t}+g(t, v) v_{x}+f(t, v)\right] \psi d x d t=0
$$


for arbitrary $\psi(t, x)$ such that $\psi, \psi_{x} \in L_{2}\left(Q_{T}\right)$. Obviously

$$
v_{t}+g(t, v) v_{x}+f(t, v)=0 \quad \text { a. e. in } Q_{T},
$$

hence from $g(t, v) v_{x}+f(t, v) \in L_{\infty}\left(Q_{T}\right)$ we conclude that $v_{t} \in L_{\infty}\left(Q_{T}\right)$.

Thus we prove the existence of the strong solution of problem (1.5)-(1.7) and prove the third part of Theorem 1 as well.

Let us turn now to the uniqueness.

Suppose that there exist two solutions $v_{1}$ and $v_{2}$. For the difference $v \equiv$ $v_{1}-v_{2}$ we have

$$
v_{t}+g\left(t, v_{1}\right) v_{x}+\left(g\left(t, v_{1}\right)-g\left(t, v_{2}\right)\right) v_{2 x}+f\left(t, v_{1}\right)-f\left(t, v_{2}\right)=0 \quad \text { a. e. in } Q_{T} \text {. }
$$

Multiply Eq. (3.6) by $v$ and integrate to obtain:

$$
\begin{aligned}
& \frac{1}{2} \frac{d}{d t} \int_{-l}^{l} v^{2} d x+\int_{-l}^{l}\left[\left(g\left(t, v_{1}\right)-g\left(t, v_{2}\right)\right) v_{2 x}+f\left(t, v_{1}\right)-f\left(t, v_{2}\right)\right]\left(v_{1}-v_{2}\right) d x \\
& \quad=\frac{1}{2} \int_{-l}^{l} g_{v_{1}}\left(t, v_{1}\right) v_{1 x} v^{2} d x .
\end{aligned}
$$

Integrate with respect to $t$. Taking into account (1.4) and the fact that $v(0, x)=$ 0 we obtain

$$
\int_{-l}^{l} v^{2} d x \leq \int_{Q_{T}} g_{v_{1}}\left(t, v_{1}\right) v_{1 x} v^{2} d x .
$$

Consequently Gronwall's inequality imply

$$
\int_{-l}^{l} v^{2} d x=0 \Leftrightarrow v \equiv 0 \quad \text { in } Q_{T} .
$$

Finally, the vanishing of $v$ as $t \rightarrow+\infty$ follows from the fact that $u_{\varepsilon_{n}} \rightarrow 0$ as $t \rightarrow 0$ and $u_{\varepsilon_{n}} \rightarrow v$ uniformly.

Remark. Actually the second part of Theorem 1 asserts that the presence of a strong damping prevents the shock formation which takes place for standard inviscid Burgers equation and for the inviscid generalized Burgers equation as well if the damping term is not strong enough. In general case there is no classical solution but there exists a unique weak solution in the sense of Kruzhkov (entropy solution), see [3] for the Cauchy problem, and [1] for the boundary value problem.

\section{On the Cauchy problem}

Consider the following Cauchy problems

$$
\begin{aligned}
u_{t}+g(t, u) u_{x}+f(t, u) & =\varepsilon u_{x x} \quad \text { in } \Pi_{T}=(0, T) \times(-\infty,+\infty), \\
u(0, x) & =\phi(x) \quad \text { for }|x|<\infty
\end{aligned}
$$


and

$$
\begin{aligned}
v_{t}+g(t, v) v_{x}+f(t, v) & =0 \quad \text { in } \Pi_{T}=(0, T) \times(-\infty,+\infty), \\
v(0, x) & =\phi(x) \text { for }|x|<\infty .
\end{aligned}
$$

Concerning the initial data $\phi(x)$ we suppose that

$$
\max _{\mathbf{R}}|\phi(x)| \leq M,|\phi(x)-\phi(y)| \leq K|x-y| \quad \forall x, y \in \mathbf{R},
$$

and in order to simplify the proof assume that

$$
\phi(x) \equiv 0 \quad \text { for }|x| \geq N
$$

where $N>0$ is some constant.

Theorem 2. Suppose that $f(t, z)$ and $g(t, z)$ are Hölder continuous functions on the set $[0, T] \times[-M, M]$ and $f(t, 0)=g(t, 0)=0$. If conditions (1.4), (4.5), (4.6) are fulfilled then

I. there exists a unique classical solution of problem (4.1), (4.2) such that

$$
\max _{\Pi_{T}}|u(t, x)| \leq M, \quad \max _{\Pi_{T}}\left|u_{x}(t, x)\right| \leq K
$$

and

$$
u(t, x) \rightarrow 0 \quad \text { as } t \rightarrow+\infty .
$$

II. there exists a strong solution of problem (4.3), (4.4) such that

$$
\max _{Q_{T}}|v(t, x)| \leq M, \quad\left\|v_{x}(t, x)\right\|_{L_{\infty}} \leq K,
$$

moreover, if $g(t, v)$ is differentiable with respect to $v$, then this solution is unique and

$$
v(t, x) \rightarrow 0 \quad \text { as } t \rightarrow+\infty
$$

III.

$$
\lim _{\varepsilon \rightarrow 0}\|u(t, x)-v(t, x)\|_{C^{0}\left(Q_{T}\right)}=0 .
$$

Proof. Let $u_{l}$ where $l \geq N$ be a unique classical solution of problem (1.1)(1.3) from Theorem 1. Notice that the estimates $\left|u_{l}\right| \leq M$ and $\left|u_{l x}\right| \leq K$ are independent of $l$. The solution of the Cauchy problem can be obtained as the limit of the sequence of solutions of problem (1.1)-(1.3) as $l \rightarrow \infty$ employing the usual diagonal process (for details see [5] Chapter V §8).

The uniqueness and the large time behavior can be proved similarly to the previous section. The second and the third part of Theorem 2 can be proved similarly to the previous section as well.

Remark. Actually condition (4.6) can be substituted by the following one

$$
\|\phi(x)\|_{L_{2}(-\infty, \infty)}<\infty .
$$




\section{On the equation $u_{t}+a u u_{x}+\lambda u=\varepsilon u_{x x}$}

In $Q_{T}$ consider equation

$$
u_{t}+a(t) u u_{x}+\lambda(t) u=\varepsilon u_{x x}
$$

without restriction (1.9) (concerning the physical meaning of this equation see $[2,12,17])$. It is clear that we can not expect to obtain uniformly with respect to $\varepsilon$ estimate of $\left|u_{x}\right|$ for arbitrary $T>0$ because the characteristics of the related hyperbolic equation intersect in finite time (see Example 1 in Introduction). However we will show that first we can obtain such estimate on $|u|$ and second we can obtain the estimate on $\left|u_{x}\right|$ independent of $\varepsilon$ for some $T^{*}>0$. Let us start from the estimate of the solution. For the problem (5.1), (1.2), (1.3) by standard procedure (see for example [5]) one can easily obtain the estimate

$$
\max _{Q_{T}}|u(t, x)| \leq M e^{\lambda_{0} T}
$$

where $\lambda_{0}=\max _{[0, T]}(-\lambda(t))$. Let us show that the estimate independent of $T$ takes place under the assumption

$$
|a(t)| \geq \alpha>0 \quad \text { for } t \in[0, T] .
$$

Lemma 5.1. Suppose that condition (5.2) is fulfilled, then for any classical solution of problem (5.1), (1.2), (1.3) the estimate

$$
\max _{Q_{T}}|u(t, x)| \leq 2 \tilde{M}=2 \max \left\{M, \frac{4 l \lambda_{1}}{\alpha}\right\}
$$

holds, here $\lambda_{1}=\max _{[0, T]}|\lambda(t)|$.

Proof. Introduce the following cut-off function

$$
g_{\tilde{M}}(z)= \begin{cases}2 \tilde{M}, & z>2 \tilde{M}, \\ z, & |z| \leq 2 \tilde{M} \\ -2 \tilde{M}, & z<-2 \tilde{M}\end{cases}
$$

Consider the auxiliary equation

$$
u_{t}+a u u_{x}=\varepsilon u_{x x}-\lambda g_{\tilde{M}}(u) \quad \text { in } Q_{T}=(0, T) \times(-l, l) .
$$

Our goal is to obtain the a priori estimate $|u| \leq 2 \tilde{M}$ for a solution of problem (5.3), (1.2), (1.3) and by this to show that Eqs. (5.3)) and (5.1) coincide which implies the same estimate for a solution of problem (5.1)), (1.2), (1.3) as well.

We start from the case $a(t, x) \geq \alpha>0$. Put

$$
w \equiv u-\frac{\tilde{M}}{2 l}(l+x)+\tilde{M}, \quad L \equiv \frac{\partial}{\partial t}-\varepsilon \frac{\partial^{2}}{\partial x^{2}} .
$$

Obviously

$$
L w=-a u u_{x}-\lambda g_{\tilde{M}}(u) .
$$


Suppose that at the point $N \in \bar{Q}_{T} \backslash \Gamma_{T}$ the function $w$ attains its positive maximum (recall that $\Gamma_{T}$ is the parabolic boundary of $Q_{T}$ ). Then at this point we have $w>0, w_{x}=0$ or $u>\tilde{M}$ and $u_{x}=\tilde{M} / 2 l$, thus $0<g_{\tilde{M}}(u)<2 \tilde{M}$ and

$$
\left.L w\right|_{N}=-a u \frac{\tilde{M}}{2 l}-\left.\lambda g_{\tilde{M}}(u)\right|_{N}<-\alpha \frac{\tilde{M}^{2}}{2 l}+\lambda 2 \tilde{M} \leq 0
$$

i. e. $\left.L w\right|_{N}<0$ which is impossible. Taking into account that $w \leq 0$ on $\Gamma_{T}$ we conclude that $w \leq 0$ in $Q_{T}$ or

$$
u \leq 2 \tilde{M} \quad \text { in } Q_{T} .
$$

Let us obtain now the estimate from the below. Consider the function

$$
\omega \equiv u+\frac{\tilde{M}}{2 l}(l-x)+\tilde{M} .
$$

Suppose that at the point $N_{1} \in \bar{Q}_{T} \backslash \Gamma_{T}$ function $\omega$ attains its negative minimum. Then at this point we have $\omega<0, \omega_{x}=0$ i.e. $u<-\tilde{M}, u_{x}=\tilde{M} / 2 l$. Thus, at this point, $-2 \tilde{M}<g_{\tilde{M}}(u)<0$ and

$$
\left.L \omega\right|_{N_{1}}=-a u \frac{\tilde{M}}{2 l}-\left.\lambda g_{\tilde{M}}(u)\right|_{N_{1}}>\alpha \frac{\tilde{M}^{2}}{2 l}+\left.\lambda_{1} g_{\tilde{M}}(u)\right|_{N_{1}} \geq \alpha \frac{\tilde{M}^{2}}{2 l}-\lambda_{1} 2 \tilde{M} \geq 0
$$

i.e. $\left.L \omega\right|_{N_{1}}>0$ which is impossible. Taking into account that $\omega \geq 0$ on $\Gamma_{T}$ we conclude that $\omega \geq 0$ in $Q_{T}$ or

$$
u \geq-\frac{\tilde{M}}{2 l}(l-x)-\tilde{M} \geq-2 \tilde{M} .
$$

Finally

$$
|u(t, x)| \leq 2 \tilde{M}
$$

Now let us turn to the case $a(t, x) \leq-\alpha$. In this case in order to obtain the estimate $u \leq 2 \tilde{M}$ instead of

$$
w \equiv u-\frac{\tilde{M}}{2 l}(l+x)+\tilde{M} \quad \text { we take } w \equiv u-\frac{\tilde{M}}{2 l}(l-x)+\tilde{M}
$$

For the establishment of the estimate from the below $u \geq-2 \tilde{M}$ instead of

$$
\omega \equiv u+\frac{\tilde{M}}{2 l}(l-x)+\tilde{M} \quad \text { we take } \omega \equiv u+\frac{\tilde{M}}{2 l}(l+x)+\tilde{M}
$$

and repeat the same procedure as in the previous case.

Lemma is proved.

Lemma 5.2. There exists $T^{*}=T^{*}\left(K, \lambda_{0}, a_{0}\right)<+\infty$ and $\nu=\nu\left(K, \lambda_{0}, a_{0}\right)$ such that for any classical solution of problem (5.1), (1.2), (1.3) the estimate

$$
\left|u_{x}(t, x)\right| \leq K e^{\nu t} \quad \text { for } t \in\left[0, T^{*}\right]
$$

holds, here $a_{0}=\max _{[0, T]}|a|$. 
Proof. Consider function $v=u e^{-\nu t}$ where positive constant $\nu>\lambda_{0}$ will be defined later. For $v$ we obtain

$$
v_{t}-\varepsilon v_{x x}=-a v v_{x} e^{\nu t}-(\lambda+\nu) v
$$

besides $v(0, x)=\phi(x)$ and $v(t, \pm l)=0$. As it follows from the proof of Lemma 2.1 (Sect. 2) if condition (1.4) for Eq. 5.4 is fulfilled then the estimate $\left|v_{x}\right| \leq K$ takes place. Condition (1.4) for Eq. (5.4) takes the following form:

$$
K|a|\left(v_{2}-v_{1}\right) e^{\nu t}<(\nu+\lambda)\left(v_{2}-v_{1}\right) \text { for } v_{2}>v_{1}
$$

or

$$
K|a| e^{\nu t}<\nu+\lambda \Leftrightarrow t<\frac{\ln \frac{\nu+\lambda}{K|a|}}{\nu} .
$$

Consider the function

$$
\psi(\xi) \equiv \frac{\ln \frac{\xi+\lambda_{0}}{K a_{0}}}{\xi}
$$

as $\nu$ we take the value of $\xi$ where the function $\psi$ obtains its maximum and put

$$
T^{*}=\frac{\ln \frac{\nu+\lambda_{0}}{K a_{0}}}{\nu} .
$$

Thus we conclude that $\left|u_{x}\right| e^{-\nu t} \leq K$ for $t \in\left[0, T^{*}\right]$.

Lemma is proved.

\section{References}

[1] Bardos, C., Leroux, A.Y., Nedelec, J.C.: First order quasilinear equations with boundary conditions. Commun. Partial Differ. Equ. 4(9), 1017-1034 (1979)

[2] Berestycki, H., Kamin, S., Sivashinsky, G.: Nonlinear dynamics and metastability in a Burgers type equation (for upward propagating flames). C. R. Acad. Sci. Paris Ser. I Math. 321(2), 185-190 (1995)

[3] Kruzhkov, S.N.: First order quasilinear equations with several independent variables. Math. Sbornik, 81(123), n.2, 228-255 (1970) (Russian). English trans. in: Math. USSR Sbornik, 10, 217-243 (1970)

[4] Kruzhkov, S.N.: Quasilinear parabolic equations and systems with two independent variables. Trudy Sem. Petrovsk. 5, 217-272 (1979) (Russian). English transl. in: Topics in Modern Math., Consultant Bureau, New York (1985)

[5] Ladyzhenskaja, O.A., Solonnikov, V.A., Uralceva, N.N.: Linear and quasilinear equations of parabolic type. (in Russian) Translated from the Russian by S. Smith. Translations of Mathematical Monographs, vol. 23 American Math. Society, Providence, R.I. 1967, 648 pp.

[6] Lardner, R.W., Arya, J.C.: Two generalizations of Burger's equation. Acta Mech. 37, 179-190 (1980) 
[7] Murray, J.D.: Perturbation effects on the decay of discontinuous solutions of nonlinear first order wave equations. SIAM J. Appl. Math. 19, 273-298 (1970)

[8] Murray, J.D.: On the Gunn effect and other physical examples of perturbed conservation equations. J. Fluid Mech. 44, 315-346 (1970)

[9] Murray, J.D.: On Burgers' model equations for turbulence. J. Fluid Mech. 59, 263-279 (1973)

[10] Rao, C.S., Sachdev, P.L., Ramaswamy, M.: Self-similar solutions of a generalized Burgers equation with nonlinear damping. Nonlinear Anal. Real World Appl. 4, 723-741 (2003)

[11] Sadchev, P.L., Nair, K.R.C., Tikekar, V.G.: Generalized Burgers equations and Euler-Painleve transcendents I. J. Math. Phys. 27(6), 1506-1522 (1986)

[12] Sun, X., Ward, M.J.: Metastability for a generalized Burgers equation with applications to propagating flame fronts. Eur. J. Appl. Math. 10(1), 27-53 (1999)

[13] Sadchev, P.L., Nair, K.R.C.: Generalized Burgers equations and Euler-Painleve transcendents II. J. Math. Phys. 28(5), 997-1004 (1987)

[14] Tersenov, Al.S., Tersenov, Ar.S.: On the Bernstein-Nagumo's condition in the theory of nonlinear parabolic equations. J. Reine Angew. Math. 572, 197-217 (2004)

[15] Tersenov, Al.S., Tersenov, Ar.S.: The Cauchy problem for a class of quasilinear parabolic equations. Ann. Mat. Pura Appl. (4) 182(3), 325-336 (2003)

[16] Vaganan, M., Kumaran, S.: Similarity solutions of the Burgers equation with linear damping. Appl. Math. Lett. 17(10), 1191-1196 (2004)

[17] Yu, J., Kevorkian, J.: Nonlinear evolution of small disturbances into roll-waves in an inclined open channel. J. Fluid. Mech. 243, 575-594 (1992)

A. S. Tersenov

Department of Mathematics, University of Crete,

71409 Heraklion, Crete, Greece

e-mail: tersenov@math.uoc.gr

and

Lavrentyev Institute of Hydrodynamics,

630090 Novosibirsk, Russia

Received: 05 October 2009.

Accepted: 27 January 2010. 